\title{
LESSONS AND NEW PERSPECTIVES: IS CONVALESCENT PLASMA THERAPY EFFECTIVE ON COVID-19 PATIENTS?
}

\section{HERNITA TAURUSTYA ${ }^{1 *}$, SRI YUNITA ${ }^{2}$, YSRAFIL YSRAFIL ${ }^{3,4}$, NUKE PRATIWI $^{5}$, ANINDITA RAHMATIAH ${ }^{5}$}

1Department of Pharmacology and Therapeutic, Faculty of Medicine and Health Sciences University of Bengkulu, Bengkulu, Indonesia, 2Department of Physiology, Faculty of Medicine and Health Sciences University of Bengkulu, Bengkulu, Indonesia, ${ }^{3}$ Department of Pharmacy, Politeknik Kesehatan Kementerian Kesehatan Gorontalo, Gorontalo, Indonesia, ${ }^{4}$ Faculty of Medicine, Universitas Palangka Raya, Palangka Raya, Indonesia, 5 Undergraduate of Medical Sciences, Faculty of Medicine and Health Sciences University of Bengkulu, Bengkulu, Indonesia Email: hernitataurustya.dr@unib.ac.id

Received: 17 Jun 2021, Revised and Accepted: 19 Oct 2021

ABSTRACT

Objective: Recently, convalescent plasma (CP) therapy has shown promising evidence in the treatment of several serious contagious diseases, including SARS-CoV, Influenza and Ebola. We conducted a systematic review to extract data about using CP treatment for COVID-19 patients and it's effectively.

Methods: The retrieval of studies was conducted according to Cochrane Collaboration and from electronic databases including PubMed, Medline, and others (medRxiv and BioRxiv). Searching of the available evidence concerning CP treatment of COVID-19 patients was conducted in journal articles published between December 2019 and October 2020. The articles were further screened based on inclusion and exclusion criteria to identify the high-quality studies for analysis.

Results: A total of $18 \mathrm{CP}$ studies were included in this review. We found variance regarding the effectiveness of $\mathrm{CP}$ in the reduction of mortality rate, length of stay, and increased discharging rate. Several findings show CP therapy is effective in increasing viral negativity, neutralizing antibodies to recipients, does not cause harmful adverse reactions and in some cases can improve clinical symptoms. This therapy is presently considered effective for generating good clinical outcomes when given early in the course of the disease.

Conclusion: The effectiveness of CP in terms of mortality, length of stay, and increased discharging patients is still debatable. However, CP therapy is effective in increasing the negativity of SARS-CoV-2 test, neutralizing antibody titer and is safe so it can be considered for COVID-19 patients. CP should not be given in the initial disease course but is recommended for the early disease course.

Keywords: Convalescent plasma, COVID-19, Effectivity, Neutralizing antibody, SARS-CoV-2, therapy

(C) 2022 The Authors. Published by Innovare Academic Sciences Pvt Ltd. This is an open access article under the CC BY license (https://creativecommons.org/licenses/by/4.0/) DOI: https://dx.doi.org/10.22159/ijap.2022v14i1.42452. Journal homepage: https://innovareacademics.in/journals/index.php/ijap

\section{INTRODUCTION}

The COVID-19 pandemic is currently a major public health concern and has become a significant and credible threat to economies around the world because the mortality and morbidity rates from this disease are still high. Coronavirus is a family of Severe Acute Respiratory Syndrome Coronavirus 2 (SARS-CoV-2) and Middle East Respiratory Syndrome-related Coronavirus (MERS-CoV) [1-6]. SARS-CoV-2 is a type of respiratory virus that can cause pneumonia in sufferers, and the first cases appeared in the city of Wuhan, China since December 2019. The World Health Organization (WHO) recently reported the virus had infected 194 million people and caused 4.16 million deaths [6-8]. The high incidence, worsening of disease, increasing death rate and the severe impacts caused by the disease have shown few signs of decreasing in most areas of the world. Accordingly, many leading scientists have proposed to change the term of the outbreak from pandemic to syndemic $[9,10]$. Aimed toward accelerated development and distribution, vaccines for the disease were rapidly passed through phase 3 clinical trials in several countries and approved under rushed research and unorthodox development protocols without any animal trials. Single therapy from Remdesivir is presently considered effective in treating people with COVID-19 with mild-moderate symptoms, but for COVID-19 sufferers with severe symptoms who use mechanical ventilation (MV) breathing aids, this current treatment is not effective in helping in recovery [11].

Convalescent therapy is considered efficient management that can be done with plasma transfusions. The use of plasma transfusions for the treatment of infectious diseases has long been used successfully. The efficacy of convalescent plasma (CP) therapy has shown conclusive evidence in the treatment of several infectious diseases that have occurred in the last few decades, such as SARS-CoV, Ebola virus, also in severe cases of Influenza, and recently in pneumonia disease caused by SARS-CoV-2 infection. While still controversial, several studies regarding the effectiveness and safety of these CP treatments have provided promising evidence in improving clinical symptoms, the negativity of viral test rates, as well as reducing discharge times and mortality while bringing some hope in the handling of COVID-19, which has continued to ravage the world [12-16].

We conducted a systematic review to extract available data on recovery and mortality from $\mathrm{CP}$ for the treatment of people with COVID-19. This study may help clinicians and scientists identify more effective therapy options based on current scientific evidence for potential treatment and better clinical management in COVID-19 patients with severe symptoms.

\section{MATERIALS AND METHODS}

\section{Study inclusion criteria}

The retrieval of studies was conducted using electronic databases (PubMed, Medline and others (medRxiv, and BioRxiv) to comprehensively identify journal articles. Using "Convalescent plasma”, "SARS-CoV-2", “COVID-19” and "Coronavirus Disease 2019", relevant articles were searched by abstract and title. The papers included were the original research reports about the effects of giving CP therapy to patients with COVID-19, including patients' discharge and length of stay, improvements in laboratory and radiological findings, viral rate, mortality outcome, clinical benefits and adverse events with study designs including randomized controlled trials, prospective and retrospective comparative cohort studies that were published in scientific journals. Removal of duplicates of identified studies was done manually.

\section{Exclusion criteria}

There were some exclusion criteria in this review to exclude the identified data from searching, which excluded reviews and guiding statements about clinical guidelines and expert consensus papers; 
case series and case reports of CP therapy either animal or in vitro cell studies; any article not available in full text; and studies not having complete data concerning treatment outcome, effectivity and safety of CP therapy.

\section{Screening, Data extraction and quality assessment}

Identified papers obtained from searching based on abstracts and titles using the keywords were screened based on the inclusion and exclusion criteria. Data from the included studies were further extracted for the following information: first author's name and year of publication, country, number of patients, diagnosing method, disease severity, age, concentration and frequency administration of $\mathrm{CP}$, and other drugs administrated and summarized in table 1 . Further, information about outcome, negativity rate of SARS-CoV-2 test, improvements in laboratory and radiological finding and adverse reactions are shown in table 1.

\section{Outcomes}

The outcomes we looked at and analyzed in the review included mortality outcome, discharge rate, length of stay after CPT therapy, improvements in laboratory and radiological findings, viral rate, mortality outcome, clinical benefits and adverse events.

\section{Reduction of risk of bias}

The assessment criteria for the journals were conducted independently by three authors to reduce the risk of bias in this systematic review. We used the Joanna Briggs Institute (JBI) Critical Appraisal Checklist for use in JBI Systematic Reviews to apply the criteria. Disagreements were discussed to reach consensus while assessing all of the selected article.

\section{RESULTS}

\section{Study Inclusion and characteristics}

Based on the search results using titles and abstracts, we obtained 483 articles about CP therapy for Coronavirus Disease 2019 in the PubMed and Medline database and 521 additional articles were identified from other sources, namely medRxiv.

A total of 18 article were included in this systematic review including 8 randomized controlled trials (RCT) [11, 17-23], 5 prospective studies [11, 17-23], 5 prospective studies [24-28], 1 nonrandomized multi-center clinical trial [29], and 4 retrospective studies [30-33]. We further summarized the data of all the studies into two tables. Table 1 shows the study characteristics and patients in each study, including location of the study, study type, number of participants, diagnosed tools, the severity of patients, age, dosage of transfusion, and other drug administration.

Outcomes of the studies, including discharging and mortality rates, length of stay, negative rate after convalescent plasma transfusion, improvement disease progression, improvements of laboratory and radiological findings and adverse events of CP therapy, are presented in table 2. From our review, all patients with COVID-19 receiving conventional plasma therapy were generally adults $(>18$ y) with a mean and median age above $48 \mathrm{y}$.

There were studies conducted in different countries worldwide. Eight of the RCTs were conducted in various countries, including one in China that was conducted by Li et al. with 103 patients with severe and life-threatening COVID-19. They compared clinical improvements of CP therapy $(n=52)$ vs standard treatment $(n=51)$ [23]. In Iraq, an RCT was conducted with 49 patients, including 21 patients in the CP therapy group vs 28 in the control group. Comorbidities between the two groups, including diabetes mellitus, hypertension, heart disease, obesity and cancer, did not differ significantly [11]. Another two RCT were conducted in India by Agarwal et al., and Bajpai among 464 and 29 patients, respectively. Agarway et al. and Bajpai conducted their studies on moderate and severe COVID-19 adult patients, respectively. The two studies each aimed to assess the effect of adding CP therapy intervention $(\mathrm{n}=235)+$ best standard of care (SOC) in the management of COVID-19 compared with the best SOC as the control arm ( $n=229)$ and aimed to compare the efficacy of CP therapy to fresh frozen plasma $[17,20]$.
Furthermore, two multicenter RCT studies were conducted by Avendaño-Solà on 81 early or mild COVID-19 patients in Spain, with 38 in the $\mathrm{CP}$ group $(\mathrm{CP}+\mathrm{SOC})$ vs 43 in the control group (received $\mathrm{SOC}$ ) and the other by Gharbharan et al. in the Netherlands. However, since the study conducted in Holland was halted prematurely after 86 patients were enrolled, the analysis was only performed on patients from that number including 43 in the SOC arm vs 43 in the CP arm $[19,22]$. The last two RCTs were randomized, open label pilot trials in Bahrain and a phase II RCT in Chile. Concerning the clinical trial in Bahrain, the study conducted by AlQahtani reported outcomes of 20 pilot trial patients who received SOC+two $200 \mathrm{ml} \mathrm{CP}$ transfusion compared to 20 patients who received routine care alone. Meanwhile, Balcells et al. assessed patient outcomes to compare the effectiveness of early CP therapy (n $=28)$ versus deferred CP therapy $(\mathrm{n}=30)$ in patients with severe COVID-19 [18, 21].

In addition to RCTs, a non-randomized multi-center clinical trial was included. A study was conducted in Iran and involved 189 severe COVID-19 patients and involved 115 in the CP group vs 74 in the control group. To eliminate the risk of bias, the patients that were included in this study had no differing ages, gender, comorbidity, nor radiological and clinical findings on admission [29]. We also included several prospective studies that were conducted in different countries. Duan et al., Erkurt et al., Salazar et al., and Olivares-Gazca, et al. did studies in China, Turkey, USA and Mexico, respectively, with $10,26,387$, and 10 patients diagnosed with COVID-19 using quantitative reverse transcription-polymerase chain reaction (qRT-PCR) who had entered the severe stage of the disease [24-27]. A prospective phase II clinical trial study comparing the effectiveness of using $\mathrm{CP}$ in 16 patients with transfusion in the early disease course versus 22 patients with late transfusion (in disease progression) was also included in this review [28].

Other data were collected from several retrospective studies of CP therapy in some other countries worldwide. They include the retrospective observational study done by Altuntas et al. in Turkey with a total of 1,776 severe or critically ill COVID-19 patients (888 in the CP group vs 888 in the control group). Those participants who were included in these two groups have characteristics that were not different in gender, age, comorbidities, chronic liver diseases, and antiviral treatment, making it easier for the researchers to analyze the outcome of $\mathrm{CP}$ [30]. Additionally, the retrospective studies of Omrani et al. with 80 patients ( 40 vs 40 ), Zeng et al. with 21 patients (6 vs 15) and Wu et al. with 27 patients all had similar subject characteristics that did not differ in gender, age, comorbidities, nor symptoms and clinical laboratory findings before transfusion, making it easier for the researchers to analyze the outcome of CP. Specifically, for the retrospective study by Wu et al., they compared the effectiveness of $\mathrm{CP}$ in early negative patients and late negative patients $(\mathrm{n}=15 \mathrm{vs} \mathrm{n}=12)$ [31-33].

\section{CPT dosage}

In the transfusion, the administration of CP therapy must be adjusted to the patient's ABO type of the recipient [23]. The optimal $\mathrm{CP}$ plasma transfusion dose that can be used varies, ranging from 200 to $600 \mathrm{ml}$. We found a single dose of $200 \mathrm{ml}$ was the minimum dose of CP transfusion [24-26]. One RCT demonstrated that this treatment approach could be based on the patient's body weight of approximately 4 to $13 \mathrm{ml} / \mathrm{kg}$ of recipient body mass and median volume was $200 \mathrm{ml}$ (IQR, 200-300 ml) per patient [23]. The administration can be repeated for the second or third transfusions if needed, both when clinical changes are seen in patients and to patients without clinical response and a persistently positive RTPCR [22]. A dosage of $400 \mathrm{ml}$ per administration was also given by some researchers to patients with severe COVID-19. However, the usual administration is at a dose of $200 \mathrm{ml}$ or $250 \mathrm{ml}$ per unit of administration and repeated once for a secondary transfusion with the same dosage ( 400 or $500 \mathrm{ml}$ as two 2 units). This is recommended by the majority of authors in our compiled study in both retrospective, prospective and RCTs. Meanwhile, the maximum dose of CP therapy as stated in the two retrospective observational studies, is $600 \mathrm{ml}[30,32]$. 
Table 1: Characteristic of patients given convalescent plasma

\begin{tabular}{|c|c|c|c|c|c|c|c|}
\hline $\begin{array}{l}\text { Auth } \\
\text { ors }\end{array}$ & Study type & $\begin{array}{l}\text { Disease } \\
\text { severity }\end{array}$ & $\begin{array}{l}\text { Age } \\
\text { (year) }\end{array}$ & $\begin{array}{l}\text { Concentration } \\
\text { and frequency } \\
\text { administration }\end{array}$ & $\begin{array}{l}\text { Other drug } \\
\text { administrated }\end{array}$ & Iral negative rate & Outcome \\
\hline [11] & $\begin{array}{l}\text { Randomize } \\
\mathrm{d} \\
\text { multicenter } \\
\text { clinical trial }\end{array}$ & Severe & $\begin{array}{l}55.6 \pm \\
17.83^{a}\end{array}$ & $\begin{array}{l}400 \mathrm{ml} \text { (was } \\
\text { given only once } \\
\text { for all of the } \\
\text { patients) }\end{array}$ & $\begin{array}{l}\text { Hydroxychloquine, } \\
\text { azithromycin, oxygen } \\
\text { therapy, } \\
\text { methylprednisolone }\end{array}$ & $\begin{array}{l}\text { Duration of infection } \\
19.33 \pm 6.90 \text { vs } \\
23.42 \pm 6.39(\mathrm{p}=0.037)\end{array}$ & $\begin{array}{l}\text { There were significant differences in recovery } \\
\text { time from critical illness ((RTCI) of CP group } \\
\text { and control group } 4.52 \pm 2.35 \mathrm{~d} \text { vs } 8.45 \pm 1.87 \mathrm{~d} \\
(<0.0001) \text {. RTCI of patients received CP from } \\
\text { IgM donor were lower than negative IgM } \\
\text { donors. } 3.18 \pm 1.4 \text { vs } 6 \pm 2.3(p=0.003) \text {. and RTCI } \\
\text { from donor with strongly IgG had lower than } \\
\text { moderately (p=0.048). Mortality rates of CP } \\
\text { group were lower than control group } 1 / 21 \\
(4.8 \%) \text { vs } 8 / 28(28.5 \%)(p<0.05) \text {. }\end{array}$ \\
\hline [29] & $\begin{array}{l}\text { Nonrandom } \\
\text { ized multi- } \\
\text { center } \\
\text { clinical trial }\end{array}$ & Severe & $\begin{array}{l}54.41 \\
\pm 13.7 \\
1^{\mathrm{a}} \mathrm{vs} \\
56.83 \\
\pm 14.9 \\
8^{\mathrm{a}}\end{array}$ & $\begin{array}{l}500 \mathrm{ml} \text { (within } 4 \\
\text { h) }\end{array}$ & $\begin{array}{l}\text { Lopinavir-Ritonavir, } \\
\text { Hydroxychloroquine } \\
\text { and anti- } \\
\text { inflammatory agent }\end{array}$ & $\begin{array}{l}\text { Negative rate of CP } \\
\text { group } 98(98.2 \%) \text { vs } \\
\text { control } 56(78.7 \%)\end{array}$ & $\begin{array}{l}\text { There were significant differences in length of } \\
\text { stay } 6.25 \pm 4.3312 .88 \pm 7.19(\mathrm{p}=0.000) \text {, } \\
\text { intubation } 7 \% \text { vs } 20.3 \%(\mathrm{p}=0.006) . \\
\text { discharged from hospital in less than } 5 \mathrm{~d} \text { after } \\
\text { transfusion between CP group versus control } \\
\text { group } 27(28.1 \%) \text { vs } 5(8.9 \%)(\mathrm{p}=0.010) \text { and } \\
\text { total discharged were } 98(98.2 \%) \text { vs } 56(78.7 \\
\%) \text {, and no difference in both groups in all- } \\
\text { cause mortality ( } p=0.09)\end{array}$ \\
\hline [25] & $\begin{array}{l}\text { Cohort } \\
\text { study }\end{array}$ & Severe & $\begin{array}{l}67.4 \pm \\
15.5^{a}\end{array}$ & $200 \mathrm{ml}$ & $\begin{array}{l}\text { Favipiravir, } \\
\text { hydroxychloroquine } \\
\text { and azithromycin }\end{array}$ & NA & $\begin{array}{l}\text { Of } 26 \text { patients included, } 20 \text { were alive and } 6 \\
\text { died after } 1 \mathrm{w} \text { of CPT }\end{array}$ \\
\hline [24] & $\begin{array}{l}\text { Prospective } \\
\text { observation } \\
\text { al study }\end{array}$ & Severe & $\begin{array}{l}52.5 \\
(\mathrm{IQR} \\
45.0- \\
59.5)^{\mathrm{b}}\end{array}$ & $200 \mathrm{ml}$ & $\begin{array}{l}\text { Arbidol, remdesivir, } \\
\text { ribavirin, peramivir, } \\
\text { cefoperazone, } \\
\text { moxifloxacin, } \\
\text { linezolid, tazobactam, } \\
\text { levofloxacin, } \\
\text { imipenem-sitastatin, } \\
\text { fluconazole, and } \\
\text { methylprednisolone. }\end{array}$ & $\begin{array}{l}\text { All patients were } \\
\text { negative for SARS-CoV- } \\
2 \text { RNA following CP } \\
\text { therapy. }\end{array}$ & $\begin{array}{l}\text { All patients in CP group were alive at the time } \\
\text { of follow-up. There were significant } \\
\text { differences in clinical outcome including three } \\
\text { of patients were discharged, seven cases seem } \\
\text { much improved status and ready for discharge } \\
\text { in CP group, while three deaths, six cases in } \\
\text { stabilized status, and one case in improvement } \\
\text { in the control group }(p<0.001)\end{array}$ \\
\hline [33] & $\begin{array}{l}\text { Retrospecti } \\
\text { ve } \\
\text { observation } \\
\text { al study }\end{array}$ & $\begin{array}{l}\text { Severe } \\
\text { (end } \\
\text { stage) }\end{array}$ & $\begin{array}{l}61.5 \\
\text { vs } 73^{b}\end{array}$ & $300 \mathrm{ml}$ & $\begin{array}{l}\text { Antiviral, antibiotics, } \\
\text { traditional chinese } \\
\text { medicine, Ig therapy, } \\
\text { and glucocorticoids }\end{array}$ & $\begin{array}{l}\text { Viral clearance was } \\
\text { higher than control } \\
\text { group } 6(100 \%) \text { vs } \\
4(26.7 \%)(p=0.004)\end{array}$ & $\begin{array}{l}\text { There were no differences of death rate in } \\
\text { both groups with } 5 \text { of } 6 \text { in CP group versus } 14 \\
\text { of } 16 \text { in control ( } p=0.18 \text { ). Survival periods of } \\
\text { CP group were longer than control }(p=0.03)\end{array}$ \\
\hline [27] & $\begin{array}{l}\text { Prospective } \\
\text { observation } \\
\text { al study }\end{array}$ & Severe & $\begin{array}{l}<30- \\
\geq 80\end{array}$ & one or two units & $\begin{array}{l}\text { Lopinavir/ritonavir, } \\
\text { remdesivir, ribavirin, } \\
\text { tocilizumab, } \\
\text { pednisone, } \\
\text { dexamethasone, } \\
\text { methylprednisolone, } \\
\text { hydrocortisone, } \\
\text { hydroxychloroquine, } \\
\text { azithromycin, }\end{array}$ & $\begin{array}{l}\text { Discharge rate } \\
98(87.5) \text { vs } 107(95.5) \\
(p=0.04)\end{array}$ & $\begin{array}{l}\text { CP Therapy were significantly decreases in } \\
\text { mortality ( } \mathrm{p}=0.047) \text {. CP group (with anti-RBD } \\
\text { IgG titer of } 1: 1350 \text { ) had lower risk of overall } \\
\text { mortality and mortality within } 28 \mathrm{~d} \text { compared } \\
\text { to control (RR, } 7.53 ; 95 \% \mathrm{CI}, 1.12-50.46 ; \\
p=0.04 \text {; and RR, } 5.92 ; 95 \% \mathrm{CI}, 0.90-38.84 ; \\
p=0.06 \text {, respectively). Transfusion within } 72 \mathrm{~h} \\
\text { and anti-RBD IgG titer of } \geq 1: 1350 \text { had lower } \\
\text { risk of mortality compared to }>72 \mathrm{~h} \text { and } \\
\text { titer }<1: 1350 \text {. Discharge rate } 98(87.5) \text { vs } \\
107(95.5)(p=0.04)\end{array}$ \\
\hline [31] & $\begin{array}{l}\text { Retrospecti } \\
\text { ve } \\
\text { observation } \\
\text { al study }\end{array}$ & Severe & $\begin{array}{l}53.5 \\
(\mathrm{IQR} \\
42- \\
60.5)^{\mathrm{b}}\end{array}$ & $400 \mathrm{ml}$ & $\begin{array}{l}\text { lopinavir-ritonavir, } \\
\text { azithromycin, } \\
\text { hydroxychloroquine } \\
\text { tocilizumab, } \\
\text { methylprednisolone, } \\
\text { mechanical } \\
\text { ventilation, }\end{array}$ & $\begin{array}{l}\text { There were differences } \\
\text { in viral clearance } \\
\text { between CP and SOC } \\
\text { group } 65 \% \text { versus } \\
55 \%,(p=0.49) .\end{array}$ & $\begin{array}{l}\text { There were no statistical differences in } \\
\text { improvements of respiratory support } \\
\text { ( } p=0.32) \text {, discharged alive from ICU within } 28 \\
d \text { and all-cause mortality at } 28 \mathrm{~d}(p>0.05) \text {. }\end{array}$ \\
\hline [23] & $\begin{array}{l}\text { Randomize } \\
\text { d Controlled } \\
\text { Trial }\end{array}$ & $\begin{array}{l}\text { Severe } \\
\text { or life } \\
\text { threaten } \\
\text { ing } \\
\text { COVID- } \\
19\end{array}$ & $\begin{array}{l}70 \\
(I Q R \\
62- \\
78))^{b}\end{array}$ & $\begin{array}{l}4 \text { to } 13 \mathrm{ml} / \mathrm{kg} \text { of } \\
\text { recipient body } \\
\text { weight (median } \\
\text { volume was } 200 \\
\text { ml (IQR, 200-300 } \\
\text { ml) each } \\
\text { patients) }\end{array}$ & $\begin{array}{l}\text { Antiviral, } \\
\text { antibacterial, Chinese } \\
\text { herbal medicine, } \\
\text { herbal medicine, } \\
\text { steroids, antifungal, } \\
\text { human } \\
\text { immunoglobulin, and } \\
\text { interferon }\end{array}$ & $\begin{array}{l}\text { There were significant } \\
\text { differences in negative } \\
\text { rate of viral in both } \\
\text { severe disease and life- } \\
\text { threatening patients in } \\
\text { CPT group vs Control } \\
90.5 \%(19 / 21) \text { vs } 41.2 \\
(7 / 17)(\mathrm{OR}, \\
13.57[95 \% \mathrm{CI}, 2.36- \\
77.95] ; p<.001) \text { and } \\
84.6(22 / 26) \text { vs } 34.8 \\
(8 / 23)(\mathrm{OR}, \\
10.31[95 \% \mathrm{CI}, 2.63- \\
40.50] ; p<.001)\end{array}$ & $\begin{array}{l}\text { - There was no significant difference in } 28 \text { - } \\
\text { day mortality }(15.7 \% \text { vs } 24.0 \% \text {; OR, } 0.65 \\
\text { [95\%CI, } 0.29-1.46] ; p=.30 \text { ) or time from } \\
\text { randomization to discharge ( } 51.0 \% \text { vs } \\
36.0 \% \text { discharged by day } 28 ; \mathrm{HR}, 1.61 \text { [ } 95 \% \mathrm{CI} \text {, } \\
0.88-2.93] ; p=.12) \text {. } \\
\text { - There were significantly improvements at } \\
\text { primary outcome at severe patients of CPT } \\
\text { group vs control group } 91.3 \%(21 / 23) \text { vs } \\
68.2 \%(15 / 22) \text { (HR, } 2.15 \text { [ } 95 \% \mathrm{CI}, 1.07-4.32] ; p \\
=.03 \text { ) but did not occur in life-threatening } \\
\text { patients }\end{array}$ \\
\hline [32] & $\begin{array}{l}\text { Retrospecti } \\
\text { ve } \\
\text { observation } \\
\text { al study }\end{array}$ & NA & $\begin{array}{l}64 \\
(\mathrm{IQR}, \\
57.0- \\
72.0)^{\mathrm{b}}\end{array}$ & $\begin{array}{l}400 \text { (IQR 200- } \\
600) \mathrm{ml}\end{array}$ & $\begin{array}{l}\text { Ribavirin, lopinavir, } \\
\text { favipiravir, mechanical } \\
\text { ventilation, broad- } \\
\text { spectrum antibiotic } \\
\text { therapy, corticoid } \\
\text { therapy, and } \\
\text { immunoglobulin } \\
\text { therapy. }\end{array}$ & & $\begin{array}{l}\text { Treatment CP had length of hospital stay and } \\
\text { interval between first transfusion and } \\
\text { discharge in early negative patients shorter } \\
\text { than with late negative patients with } \\
\text { prolonged positivity of SARS-CoV-2 RNA. Early } \\
\text { negative had a lower mortality rate than late } \\
\text { positive } 0(0 \%) \text { vs } 3(25 \%) \text {. }\end{array}$ \\
\hline [26] & $\begin{array}{l}\text { Prospective, } \\
\text { longitudinal } \\
\text {, single-arm, } \\
\text { and quasi }\end{array}$ & Severe & $\begin{array}{l}53 \\
\text { (range } \\
27- \\
72)^{\mathrm{b}}\end{array}$ & $200 \mathrm{ml}$ & $\begin{array}{l}\text { Lopinavir/ritonavir, } \\
\text { azithromycin, } \\
\text { tocilizumab, } \\
\text { hydroxychloroquine, }\end{array}$ & $\begin{array}{l}\text { Giving CP in early } \\
\text { negative patients had } \\
\text { significantly decrease } \\
\text { viral load compare late }\end{array}$ & $\begin{array}{l}\text { There was a significant decreasing of SOFA } \\
\text { score in } 8 \mathrm{~d} \text { therapy from } 3 \text { to } 1.5(\mathrm{p}=0.014) \text {, } \\
\text { increasing Kirby index }(\mathrm{PaO} 2 / \mathrm{FiO}) \text { score } \\
\text { from } 124 \text { to } 255(\mathrm{p}<0.0001) \text {, Overall survival }\end{array}$ \\
\hline
\end{tabular}




\begin{tabular}{|c|c|c|c|c|c|c|c|}
\hline $\begin{array}{l}\text { Auth } \\
\text { ors }\end{array}$ & Study type & $\begin{array}{l}\text { Disease } \\
\text { severity }\end{array}$ & $\begin{array}{l}\text { Age } \\
\text { (year) }\end{array}$ & $\begin{array}{l}\text { Concentration } \\
\text { and frequency } \\
\text { administration }\end{array}$ & $\begin{array}{l}\text { Other drug } \\
\text { administrated }\end{array}$ & Iral negative rate & Outcome \\
\hline & $\begin{array}{l}\text { experiment } \\
\text { al }\end{array}$ & & & & steroids & $\begin{array}{l}\text { negative of prolonged } \\
\text { positivity of SARS-CoV- } \\
2 \text { RNA at days } 3,5 \text { and } \\
7 \text { post-transfusion } \\
(p<0.05)\end{array}$ & $\begin{array}{l}\text { of patient was } 77 \% \text { in } 24 \mathrm{~d} \text { after CPT, } 5 \text { on } \\
\text { mechanical ventilation were extubated and } \\
\text { only two patients were dead }\end{array}$ \\
\hline$[17]$ & $\begin{array}{l}\text { Randomize } \\
\text { d controlled } \\
\text { trial }\end{array}$ & $\begin{array}{l}\text { Moderat } \\
\mathrm{e}\end{array}$ & $\begin{array}{l}52 \\
(\mathrm{IQR} \\
41 \text { and } \\
42- \\
60)^{\mathrm{b}}\end{array}$ & $200 \mathrm{ml}$ in twice & $\begin{array}{l}\text { remdesivir, } \\
\text { lopinavir/ritonavir, } \\
\text { oseltamivir, } \\
\text { hydroxychloroquine, } \\
\text { spectrum antibiotics, } \\
\text { steroids, and } \\
\text { tocilizumab }\end{array}$ & NA & $\begin{array}{l}\text { There was higher improved resolution of } \\
\text { shortness of breath and fatigue in the intervention } \\
\text { arm. There was no significant difference in WHO } \\
\text { ordinal scale scores and reduction in progression } \\
\text { to severe, mechanical ventilation, and all-cause } \\
\text { mortality of patients at } 28 \mathrm{~d} \text { after CP therapy with } \\
\text { the risk difference } 0.008 \text { ( } 95 \% \text { confidence } \\
\text { interval }-0.02 \text { to } 0.078 \text { ); risk ratio } 1.04,95 \% \\
\text { confidence interval } 0.71 \text { to } 1.54 \text { compared to } \\
\text { control arm. }\end{array}$ \\
\hline [19] & $\begin{array}{l}\text { Randomize } \\
\text { d controlled } \\
\text { trial }\end{array}$ & Mild & $59 \mathrm{~b}$ & $250-300 \mathrm{ml}$ & No Mentioned detail & $\begin{array}{l}\text { There was a significant } \\
\text { difference of viral } \\
\text { clearance at day } 7 \text { with } \\
117 / 173(68 \%) \text { in } \\
\text { intervention arm versus } \\
93 / 169(55 \%) \text { (RR } 1.2 \\
(1.04 \text { to } 1.5) \text { of control } \\
\text { arm. However, at day } 3 \text { it } \\
\text { was } 79 / 184(43 \%) \\
\text { versus } 67 / 183(37 \%) \\
1.2(\operatorname{RR}(0.9 \text { to } 1.5)) .\end{array}$ & $\begin{array}{l}\text { Progression of CPT patient were lower than } \\
\text { SOC ( } 0 \text { of } 38 ; 0 \% \text { vs } 7 \text { of } 43 \text { patients, } 14 \% \\
\text { ( } p=0.03) \text {, mortality rates were } 0 \% \text { vs } 9.3 \% \text { at } \\
\text { days } 15 \text { and } 29 \text { for CPT. There was no } \\
\text { significant in overall survival ( } p=0.06) \text {, first } \\
\text { clinical deterioration }(p=0.07) \text {, discharging } \\
\text { duration, and time of improvements. }\end{array}$ \\
\hline [22] & $\begin{array}{l}\text { Randomize } \\
\text { d controlled } \\
\text { trial }\end{array}$ & $\begin{array}{l}\text { mild } \\
\text { and } \\
\text { moderat } \\
\text { e }\end{array}$ & $\begin{array}{l}63 \\
(\mathrm{IQR} \\
56- \\
74)^{\mathrm{b}}\end{array}$ & $\begin{array}{l}300 \mathrm{ml} \text { and can } \\
\text { be repeated if } \\
\text { needed }\end{array}$ & $\begin{array}{l}\text { chloroquine, } \\
\text { azithromycin, } \\
\text { lopinavir/ritonavir, } \\
\text { tocilizumab, and } \\
\text { anakinra }\end{array}$ & $\begin{array}{l}\text { viral negativity rate } \\
\text { was } 79.7 \% \text { in CP group } \\
\text { versus } 66.54 \% \text { in } \\
\text { control at } 29 \mathrm{~d}\end{array}$ & $\begin{array}{l}\text { There was no significantly difference in overall } \\
\text { mortality (CI } 0.20-4.67, \mathrm{p}=0.95 \text { ), time to } \\
\text { discharge (HR } 0.88 \text { CI } 0.49 ; 1.60, p=0.68 \text { ) or } \\
\text { day-15 disease severity ( } \mathrm{p}=0.58 \text { ) was } \\
\text { observed in both of CPT and SOC }\end{array}$ \\
\hline [21] & $\begin{array}{l}\text { Randomize } \\
\text { d controlled } \\
\text { trial }\end{array}$ & Severe & $\begin{array}{l}65.8 \\
(27- \\
92)^{\mathrm{a}}\end{array}$ & $\begin{array}{l}400 \mathrm{ml} \text { as two } \\
200 \mathrm{ml} \text { unit }\end{array}$ & $\begin{array}{l}\text { Lopinavir/ritonavir, } \\
\text { hydroxychloroquine, } \\
\text { tocilizumab, Steroids, } \\
\text { Thromboprophylaxis, } \\
\text { and Anticoagulation }\end{array}$ & NA & $\begin{array}{l}\text { There were no significant differences in both } \\
\text { groups of early and deferred therapy of CP in } \\
\text { primary outcome } 32.1 \%(9 / 28) \text { vs } 33.3 \% \\
(10 / 30) \text { (OR } 0.95,95 \% \text { CI } 0.32-2.84) \text {. They are } \\
\text { including death in hospital }(5 / 28) \text { vs } 6.7 \% \\
(2 / 30) \text { (OR } 3.04,95 \% \text { CI } 0.54-17.2) \text {, } \\
\text { mechanical ventilation } 17.9 \%(5 / 28) \text { vs } 6.7 \% \\
(2 / 30) \text { (OR } 3.04,95 \% \text { CI } 0.54-17.2) \text {, and } \\
\text { hospitalization }>14 \text { d } 21.4 \%(6 / 28) \text { vs } 30 \% \\
(9 / 30) \text { (OR } 0.64,95 \% \text { CI } 0.19-2.1)\end{array}$ \\
\hline [18] & $\begin{array}{l}\text { Randomize } \\
\text { d controlled } \\
\text { trial }\end{array}$ & $\begin{array}{l}\text { severe } \\
\text { and/or } \\
\text { life- } \\
\text { threaten } \\
\text { ing }\end{array}$ & $\begin{array}{l}52.6 \\
(14.9) \\
\text { vs } \\
50.7 \\
(12.5)^{\text {a }}\end{array}$ & $\begin{array}{l}400 \mathrm{ml} \text { as two } \\
200 \mathrm{ml} \text { unit }\end{array}$ & $\begin{array}{l}\text { Lopinavir/ritonavir, } \\
\text { Ribavirin, } \\
\text { Hydroxychloroquine, } \\
\text { Azithromycin, } \\
\text { Peginterferon, } \\
\text { tocilizumab, Methyl } \\
\text { Prednisolone, } \\
\text { Antibiotics, } \\
\text { Anticoagulation }\end{array}$ & $\begin{array}{l}\text { Rate of negative both } \\
\text { of group early and } \\
\text { deferred therapy of CP } \\
\text { on day } 3(26 \% \text { vs } 8 \% \text {, } \\
p=0.20) \text { nor on day } 7 \\
(38 \% \text { vs } 19 \%, p=0.37)\end{array}$ & $\begin{array}{l}\text { There were no significant differences on } \\
\text { ventilation time } 10.5 \mathrm{~d} \text { vs } 8.2(\mathrm{P}>0.81) \text { and } \\
\text { discharge alive ( } 19 \text { vs } 18) \text {, total death } 1 \text { vs } 2 \\
\text { both of CPT and dead }\end{array}$ \\
\hline [20] & $\begin{array}{l}\text { Randomize } \\
\text { d controlled } \\
\text { trial }\end{array}$ & Severe & $\begin{array}{l}48.2 \pm \\
9.8^{\mathrm{a}}\end{array}$ & $\begin{array}{l}500 \mathrm{ml} \text { as two } \\
\text { unit }\end{array}$ & $\begin{array}{l}\text { Hydroxychloroquine, } \\
\text { Azithromycin, }\end{array}$ & NA & $\begin{array}{l}\text { There were no significant improvements in both } \\
\text { of CPT and FFP including needed of mechanical } \\
\text { ventilation }(\mathrm{p}=0.26 \text { ), mortality rate, ICU stay and } \\
\text { Vasopressors requirement till } 28 \mathrm{~d} \text {. CPT showed } \\
\text { significant benefits in the secondary outcome of } \\
\text { this research, including reduction of respiratory } \\
\text { rate per min [ } \mathrm{p}=0.004] \text { and }[\mathrm{p}=0.008], 02 \\
\text { saturation } \mathrm{p}<0.001 \text { and } \mathrm{p}=0.026, \mathrm{SOFA} \mathrm{p}=0.01 \\
\text { and } \mathrm{p}=0.04, \text { improvements of } \mathrm{PaO} 2 / \mathrm{FiO} 2 \mathrm{p}=0.009 \\
\text { and } \mathrm{p}=<0.001 \text { at } 48 \mathrm{~h} \text { and at day } 7 \text { respectively. }\end{array}$ \\
\hline [30] & $\begin{array}{l}\text { Retrospecti } \\
\text { ve } \\
\text { observation } \\
\text { al study }\end{array}$ & $\begin{array}{l}\text { severe } \\
\text { or } \\
\text { critically }\end{array}$ & $\begin{array}{l}60(19 \\
\pm 96)^{\mathrm{b}} \\
\text { vs } \\
61(21 \\
\pm 91)^{\mathrm{b}}\end{array}$ & $200-600 \mathrm{ml}$ & $\begin{array}{l}\text { favipiravir, } \\
\text { lopinavir+ritonavir, } \\
\text { hydroxychloroquine, } \\
\text { high dose vitamin C, } \\
\text { azithromycin }\end{array}$ & $\begin{array}{l}\text { There was no } \\
\text { significantly difference } \\
\text { in improvements of Ct } \\
\text { value at } 7 \mathrm{~d} \text { both of } \\
\text { group CPT and FFP }\end{array}$ & $\begin{array}{l}\text { CPT could reduce time in ICU, rate of MV } \\
\text { support and vasopressor support than control } \\
\text { group }(p=0.001, p=0.02, p=0.001) \text {. Although } \\
\text { CFR of CP group was lower than control } 24.7 \\
\% \text { vs } 27.7 \% \text {, but it was not statistically } \\
\text { significant }(p=0.150) \text {, the same with duration } \\
\text { in hospital. Administration of CP } 20 \mathrm{~d} \text { after } \\
\text { diagnosis of COVID-19 increases the rate of } \\
\text { MV support more than when administrated in } \\
\leq 5 \mathrm{~d}, 6-10 \mathrm{~d}, 11-15 \mathrm{~d})(p=0.001)\end{array}$ \\
\hline [28] & $\begin{array}{l}\text { A } \\
\text { Prospective } \\
\text { Phase II } \\
\text { Clinical } \\
\text { Trial }\end{array}$ & $\begin{array}{l}\text { moderat } \\
\text { e, severe } \\
\text { and } \\
\text { critically }\end{array}$ & $\begin{array}{l}63 \\
(12)^{\mathrm{a}}\end{array}$ & $\begin{array}{l}200 \mathrm{ml} \text { or } 400 \mathrm{ml} \\
\text { as two } 200 \mathrm{ml} \\
\text { unit }\end{array}$ & $\begin{array}{l}\text { Renal replacement } \\
\text { therapy, Antibiotics, } \\
\text { Antifungals, } \\
\text { Azithromycin, } \\
\text { Hydroxychloroquine, } \\
\text { IL-6 Inhibitors, } \\
\text { Remdesivir, } \\
\text { Vasopressors, Steroids, } \\
\text { Anticoagulants, and } \\
\text { Zinc }\end{array}$ & NA & $\begin{array}{l}\text { Giving CP therapy early in the disease course } \\
\text { as compare with late administration when the } \\
\text { disease of patients had progress had } \\
\text { significantly lower mean hospital length of } \\
\text { stay } 15.4 \text { vs } 33 \mathrm{~d}(p<0.01) \text { and shorter hospital } \\
\text { mortality } 13 \% \text { vs } 55 \%(p<0.02) \text {. }\end{array}$ \\
\hline
\end{tabular}


Other therapy (Antiviral, antifungal/antibiotic, steroid drug and others)

Hydroxychloquine (HCQ) or chloquine (CQ) is one drug that is almost always used in COVID-19 therapy as SOC as recommended by the WHO in the COVID-19 Treatment Guidelines. In these guidelines, it is recommended that the drug is administrated at a dose of 800 $\mathrm{mg}$ of HCQ or $1 \mathrm{~g} \mathrm{CQ}$ PO in $1 \mathrm{~d}$ then HCQ $400 \mathrm{mg}$ or $500 \mathrm{mg}$ CQ PO once daily for 4-7 d of total treatment based on clinical evaluation. Together with the two quinone drugs, the antiviral combination of lopinavir/ritonavir (LPV/RTV) is rarely absent in any therapy for COVID-19 patients in our compiled studies. Based on the guidelines issued by the WHO, the recommended dosage of LPV $400 \mathrm{mg} / \mathrm{RTV}$ $100 \mathrm{mg}$ PO is given twice daily for 10-14 d in adults. Other antiviral drugs that we found used in the treatment of the disease symptoms caused by the SARS-CoV-2 virus are ribavirin, remdesivir, oseltamivir, favipiravir, and peramivir $[17,24,25,29]$.

Antibiotic and anti-inflammatory drugs are also the most commonly prescribed therapy for COVID-19 patients, including azithromycin, cefoperazone, moxifloxacin, linezolid, tazobactam, levofloxacin, imipenem-cytastatin, broad-spectrum antibiotics, dexamethasone, methylprednisolone, hydrocortazole, and anakinra antifunctions. Monoclonal antibodies are also frequently prescribed in the treatment of pneumonia caused by the novel coronavirus, such as tocilizumab and peginterferon. Other adjunctive drugs such as traditional Chinese medicine, anticoagulants and vasopressors have been reported in some studies as effective treatment regimens for the disease [11, 18, 22-24, 27, 28, 32].

\section{Improvements in laboratory and radiological findings}

Improvement in laboratory and radiological findings is one of the important aspects measured after COVID-19 treatment as parameters of the effectiveness of CP treatment in patients (table 1). Most of the studies we compiled indicated that IgG and IgM titers increased post-transfusion with $\mathrm{CP}$. The immunoglobulin is a neutralizing antibody that comes from donor plasma that has previously been formed due to exposure to the SARS-CoV-2 virus. This increase in immunoglobulin levels was also followed by several other clinical laboratory findings that are considered markers of improvement in the patient's condition. From several studies that we analyzed, the other laboratory parameters, which we found changed from the baseline condition when the patient was treated, included decreased hemoglobin levels, increased lymphocyte counts, decreased C-reactive protein (CRP), alanine aminotransferase (ALT), and aspartate aminotransferase (AST), as well as changes (delta) in ferritin and fibrinogen [24, 27].

The clinical laboratory change in serological components was found to be associated with improved radiological findings as described in the study conducted by Duan et al. that found CP generated increased lymphocyte counts, as well as decreased CRP, ALT, and AST. These were associated with improvement in pulmonary infiltrates, gradual absorption of lung lesions and disappearing massive infiltration and ground-glass attenuation following CP therapy [24]. A prospective single-arm study conducted by OlivaresGazca et al. in 2020 also demonstrated that there were decreasing of body temperature $38.1^{\circ} \mathrm{C}$ to $36.9^{\circ} \mathrm{C}(p=0.0058)$ and serum ferritin $(p<0.05)$ after patients received treatment. Even though there were no significant differences in CRP and D-dimer levels, improvements were found in both chest X-rays of 7 of 10 patients and computerized tomography (CT) scans showing improvements of lung injury post-therapy [23].

However, several laboratory parameters such as levels of CRP, inflammatory cytokines: Interleukin (IL)-1 $\beta$, IL-6, IL-10 and tumor necrosis factor (TNF)- $\alpha$ ), D-dimer, lymphocyte count, lactate dehydrogenase (LDH), procalcitonin, ALT, and AST, which most studies included in the review that demonstrated CP therapy was not significantly different from SOC [17, 20, 25, 27, 31]. For example, the randomized controlled study by AlQahtani et al. in 2020 found there were no significant differences of laboratory findings in both the CPT group and controls in white blood cell (WBC), LDH, troponin, D-Dimer and procalcitonin levels. The same results were demonstrated by Balcells et al. in their RCT. They found there were no differences between study groups (CP vs control group) in levels of CRP ( $p=0.39$ and 0.94$)$, IL-6 ( $p=0.86$ and 1.00$)$, ferritin $(p=0.78$ and 0.92), LDH ( $p=0.78)$ and 0.58), D-dimer (0.87 and 0.68), procalcitonin $(p=0.82$ and 0.96$)$ nor lymphocyte count $(p=0.15$ and 0.66$)$ at days 3 and $7[18,21]$.

\section{Viral negative rate}

Viral load is one indication of the severity and progression of the disease caused by SARS-CoV-2, which is tested before and after treatment [34]. Administration of CP containing neutralizing antibodies is expected to reduce the amount of the virus, relieve symptoms and even cure infected patients. Some studies always include this parameter to assess the effectiveness of therapy. Agarwal et al. in their RCT found no statistically significant difference between the $\mathrm{CP}$ arms vs the SOC arm in negative viral rate with $79 / 184(43 \%)$ versus $67 / 183$ (37\%) 1.2 (RR (0.9 to 1.5)) and $117 / 173(68 \%)$ versus 93/169 (55\%) (RR 1.2 (1.04 to 1.5) at both days 3 and 7 after transfusion. The similar RCT finding was found in a study conducted by Balcells et al. on the same day post-transfusion showing that the percentage for the negative rate of patients receiving early and deferred therapy did not differ $(26 \%$ vs $8 \%, p=$ 0.20 ) in day 3 nor on day 7 ( $38 \%$ vs $19 \%, p=0.37$ ). This finding was also demonstrated in the pilot RCT results by Bajpai et al. in severely ill COVID-19 patients post $7 \mathrm{~d}$ CP therapy versus fresh frozen plasma.

Another finding by Wu et al. in 2020 demonstrated that patients given CP therapy had significantly reduced viral load in early negative compared to late negative in days 3, 5 or 7 after transfusion. An RCT conducted by Li et al. also stated that the administration of CP therapy led to higher negative rates of SARS-CoV-2 compared with the SOC at $72 \mathrm{~h}$ with a percentage $87.2 \%$ vs $37.5 \%$ (OR, 11.39 [95\% CI, 3.9133.18]; $p<0.001$ ). The studies conducted by Avendaño-Solà et al. and Zeng et al. also found that CP can increase the clearance of SARS-CoV-2 higher than the SOC at $29 \mathrm{~d}$ after therapy $(79.7 \%$ vs $66.54 \%)$ with 6 (100) vs. 4 (26.7) ( $p=0.004)$, respectively. Similarly, one RCT found that CP therapy could significantly reduce infection duration when compared with standard therapy with a mean of $19.33 \pm 6.90$ vs $23.42 \pm 6.39(p=0.037)$.

\section{Clinical benefits, length of stay and patients' discharge after CP therapy}

Concerning these outcome parameters, we found a variance of the findings in the studies that we compiled. Several studies demonstrated that adding CP therapy to the SOC for COVID-19 patients was effective in increasing recovery time from critical illness, reducing time in the intensive care unit (ICU), rate of reducing mechanical ventilator (MV) support, with lesser length of stay and vasopressor support than the control group $[11,26,29,30]$. One RCT showed that there were significantly improved primary outcomes of severe patients of the CP treatment group compared to the control group with $91.3 \%(21 / 23)$ vs $68.2 \%(15 / 22)$ (HR, 2.15 [95\% CI: 1.07-4.32]; $p=0.03$ ) at $28 \mathrm{~d}$. Although the rate of discharging and mortality did not differ significantly between the two arms, however, this study showed a positive result in patients receiving this therapy [23].

The administration of CP therapy also generated an increase in discharging rate within $5 \mathrm{~d}$, which was associated with an increase in the negativity rate of the SARS-CoV-2 RNA test when compared to the control group [29]. The association of better results with the use of CP compared to the SOC in the discharge rate of patients was also demonstrated by the studies conducted by both Duan et al. and Salazar et al. $[24,27]$. Wu et al. recommended that this therapy be done in the early disease course because giving $\mathrm{CP}$ at that time is more effective in reducing the length of hospital stay and the interval between first transfusion and improving patient discharge time when compared to late negative patients [32]. The same result was demonstrated by Ibrahim et al. that recommended that this therapy should be given at the beginning of the disease course to achieve the desired effect, which cannot be separated from their findings that there was a significantly lower mean hospital length of stay 15.4 vs 33 . days $(p<0.01)$ when compared to giving CP after the disease has progressed while extending the survival period when 
compared to the control group $(p=0.03)[28,33]$. In addition to some of the clinical effects mentioned above, other clinical benefits reported by patients after this therapy include a significant decreasing of Sequential Organ Failure Assessment (SOFA) score, decreasing patient disease progression, increasing Kirby index (PaO2/FiO2) score and improving the resolution of shortness of breath and fatigue in the intervention arm $[17,19,26]$.

Contrary to these results, a retrospective study involving 1,776 moderate, severe and critically ill COVID-19 patients concluded that the administration of CP therapy could not change the CFR or duration in hospital of patients when compared to the SOC of $24.7 \%$ or 219 patients in the CP group vs $27.7 \%$ or 246 patients in the control group ( $p=0.150)$ [30]. The same finding was stated by Gharbharan et al. in 2020, demonstrating there was no difference inhospital stay $(p=0.68)$ nor day-15 disease severity $(p=0.58)$ observed between CP-treated patients and SOC, nor in time to discharge [22]. The absence of association between CP and length of stay, discharge and mortality rate day was also found in several other studies that we included in our review $[17,23,33]$.

\section{Mortality outcome}

The effectiveness of CP therapy is also assessed by its ability to reduce mortality in patients diagnosed with pneumonia-related SAR-CoV-2 infection. Several studies have reported that this therapy is not effective in reducing patient mortality, especially in the severe stage. For example, the nonrandomized multicenter clinical trial by Omrani et al. as well as several RCT studies conducted by researchers in China, India and the Netherlands stated that adding this therapy into the SOC was not effective enough in reducing the mortality rate of severe COVID-19 patients when compared to the SOC alone (table 2) [17, 20, 22, 23, 31, 32].

However, several other studies refute these findings. For example, an RCT conducted by Rasheed et al. stated that CP therapy was able to reduce the mortality rate of patients when compared to controls in this case the SOC $[25,27]$. Other findings also confirm the positive effect of $\mathrm{CP}$ and provide recommendations regarding deciding the timing of CP transfusion given to patients. The authors found that this therapy generates $77 \%$ higher overall survival rates in patients and also confirms that CP therapy in the early disease course had lower hospital mortality of $13 \%$ vs $55 \%(p<0.02)$ when compared to disease progress in untreated patients. Accordingly, they recommended that therapy be given in the early disease course $[26,28,32,33]$.

\section{Adverse events}

There were no serious adverse events associated with CP therapy in most of the included studies. Some of the side effects that appeared in patients are mild allergic reactions, evanescent facial red spots and one had transient transfusion reactions. However, they are very rare and usually improve before the therapy ends [11, 24, 30]. Mild allergic reactions involved the development of skin redness and itching lasted for one hour after receiving $\mathrm{CP}$ and subsequent injected intramuscular antihistamine terminated the allergic cutaneous manifestations [11]. Reports of minor side effects also came from a RCT of 464 patients in India that reported similar events, namely the findings of a voluntary intervention group had minor adverse events of pain at the infusion site, chills, nausea, bradycardia, and dizziness, while 3 patients reported fever and tachycardia and 2 each had dyspnea and blockage of an intravenous catheter [17]. Additionally, a pilot RCT reported a case of mild urticaria in both the control and CP arms [20].

Two other studies involving 81 and 58 patients, respectively, also reported a small proportion of their participants suffered from side effects, namely 2 patients with suspected TRALI who gradually recovered before the study was done and 3 with fever, 1 rash, 3 serious adverse events $(2$ developed to severe respiratory deterioration within $<6 \mathrm{~h}$, and 1 TRALI type II). One of the patients later developed severe thrombocytopenia within $48 \mathrm{~h}$ post-transfusion $[19,21]$.

\section{DISCUSSION}

The highly varied findings from the existing CP therapies that we included in this review make it difficult for the researchers to determine whether these therapies are effective in curing COVID-19 patients. Several studies stated that this therapy could not reduce the mortality rate when compared with the SOC, but some also stated that this therapy could reduce the length of stay, discharge time and relieve clinical symptoms of patients, such as increasing the rate of viral negativity, with improvements in clinical and radiological findings.

The most common outcome found was an increase in the viral negativity rate of post-receiving CP patients compared to the SOC group. This negative rate cannot be separated from the role of neutralizing antibodies in donor plasma which is transfused into sick patients. The presence of Nabs is crucial in viral clearance and is associated with the efficacy of this therapy. An RCT conducted by Bajpai demonstrated the presence of a significantly increasing of S1 RBD IgG antibody titer post-transfusion $(p=<0.001)$. Furthermore, the study conducted by Rasheed demonstrated that an increase in the level of neutralizing antibody was associated with a decrease in the duration of viral infection. The antibodies in the transfused plasma bind to the receptor-binding domain of SARS-CoV-2 and prevent the virus from attacking the ACE2 receptors [35].

The SARS-CoV-2 antibodies that can bind to the SARS-CoV-2 are generally IgM, IgG1, IgG3, and IgA. In the binding of Nabs-virus, antibodies will recognize the virus and activate the antiviral effector of innate immune cells. The Fab region of an antibody will bind to $\mathrm{Fc} \gamma$ receptors of NK cells and trigger antiviral activation to eradicate viruses or virus-infected cells through the induction of antibodydependent cellular cytotoxicity. The presence of these antibodies will also bind to FcR of macrophages and trigger phagocytosis. Two key antibodies, IgG1 and 3, are opsonin molecules that can bind directly to SARS-CoV-2. The binding of IgG1 and 3-SARS-CoV-2 will also generate opsonophagocytosis of virus particles by plasmacytoid dendritic cells and conventional dendritic cells and activate responses directly and/or via NK cells and T cells [36, 37]. The mechanism might explain the high negativity rate of patients after convalescent plasma transfusion. Therefore, it is important to consider the timing of the plasma collection as well as the symptoms of the donor to ensure a high antibody titer is effective when transfused into a patient. Li et al. recommended that retrieval be done at $28 \mathrm{~d}$ post-onset of symptoms in recovery for COVID-19 patients with a history of fever with more than $38.5^{\circ} \mathrm{C}$ of body temperature longer than $3 \mathrm{~d}$. This is based on their findings that at that time, the S-RBD-specific IgG antibody levels were higher to donate $[13,38]$. CP transfusion is highly recommended in the early of disease course. Administration of CP therapy in recent symptoms of onset can be effective in reducing the mortality rate compared with late transfusion [27, 28]. Early transfusion allows for an increase of the level and binding ability of IgG and generates improvement to humoral immune responses, prevents unwanted immune responses, avoids the cytokine storm, and prevents worsening of the patient's disease condition to a critical stage [13].

In terms of the dose of transfusion, although $\mathrm{CP}$ transfusion appears to be safe because there have not been any serious adverse events in most of the studies, transfusion dosing needs to be done carefully. In the articles we included in this review, CP transfusion is recommended to be done per $200-400 \mathrm{ml}$ for each administration and can be repeated if the patient has not shown signs of improvement or is still a positive SARS-CoV-2 test. They also suggest $600 \mathrm{ml}$ as the highest dose to avoid the side effects of this CP, since it is known that, apart from neutralizing antibodies, in the plasma received from donor patients, there are several other products such as pro-inflammatory cytokines, clotting factors, defensins, and pentraxins. Excessive presence of pro-inflammatory proteins such as IL-1 $\beta$, IL-2, IL-6, IL-17, IL-8, TNF $\alpha$ and CCL2 may indicate worsening of the cytokine storm and generate pulmonary damage, and decreasing of pulmonary capacity $[13,39]$.

Increased viral negative rates and clinical finding have been associated with reduction of length of hospital stay, mortality rate, increased discharging rate and reduction of recovery time. The RCT by $\mathrm{Li}$ et al. found that an increase in negative viral rate was associated with significant improvements to the primary outcome in severe COVID-19 patients. The same result was also found by 
several other researches that showed this therapy was able to decrease the duration of the infection and increase the negative test result of the patients causing them to be discharged from the hospital faster, increasing their time to recovery and recovery rate and reducing the mortality rate $[11,23,29]$.

These results cannot fully generalize the findings that adding CP therapy to the SOC is effective in improving the clinical mortality, discharging rates, improvements of the clinical finding of patients. This is because contrarily, there were findings of existing studies that have stated that there are no significant differences between CP and SOC while other studies claim CP to be effective comparing it to the SOC alone. However, this therapy is worth considering because plasma transfusion is generally not associated with any adverse reaction events. Further research on a large scale and with a better design is needed to assess the effectiveness of this therapy and confirm these findings.

\section{CONCLUSION}

In summary, the results of this review related to the effectiveness of convalescent plasma cannot be completely concluded to apply in the general population and it is necessary to conduct RCT research on a larger scale. The studies that we included in this review have various conclusions regarding the effectiveness of convalescent plasma. Although the majority of RCTs state that CP does not reduce mortality and increase discharging rate, it is effective in increasing viral negativity and the Nabs titer. Most RCTs and several other studies stated that this therapy can increase recovery time, negativity rate, discharging rate and survival period. These findings provide evidence that this therapy needs to be considered in the management of COVID19 patients, given that there is no therapy that effectively treats the diseases caused by SARS-CoV-2 infection. Moreover, according to the data we included in this study, most found that this therapy is safe to use and does not cause any serious adverse reactions that endanger users. In addition, from the results of the various studies, they recommended that plasma collection from donors be done from the appropriate donor, namely from recovered COVID-19 patients within the $28 \mathrm{~d}$ period post-onset of symptoms with a history of fever of more than $38.5^{\circ} \mathrm{C}$ of body temperature longer than $3 \mathrm{~d}$. The convalescent plasma treatment is recommended to be done in the early disease course for maximum therapeutic effect.

\section{ACKNOWLEDGEMENT}

The authors would like to thank the support from the Faculty of Medicine and Health Sciences University of Bengkulu.

\section{FUNDING}

Nil

\section{AUTHORS CONTRIBUTIONS}

All authors have contributed equally.

\section{CONFLICT OF INTERESTS}

The authors declare that there are no conflicts of interests.

\section{REFERENCES}

1. Astuti I, Ysrafil. Severe acute respiratory syndrome coronavirus 2 (SARS-CoV-2): an overview of viral structure and host response. Diabetes Metab Syndr. 2020;14(4):407-12. doi: 10.1016/j.dsx.2020.04.020, PMID 32335367.

2. Ysrafil Y, Astuti I, Mus R, Gama NI, Rahmaisyah D, Nur'amalia R. A summary of coronavirus disease 2019: what we should know? Pharm Sci. 2020;26:S24-S35:S24-35. doi: 10.34172/PS.2020.82.

3. Viscusi WK. Pricing the global health risks of the COVID-19 pandemic. J Risk Uncertain. 2020:1-28. doi: 10.1007/s11166020-09337-2, PMID 33162671.

4. Pak A, Adegboye OA, Adekunle AI, Rahman KM, McBryde ES, Eisen DP. Economic consequences of the COVID-19 outbreak: the need for epidemic preparedness. Front Public Health. 2020;8:241. doi: 10.3389/fpubh.2020.00241, PMID 32574307.

5. Rizkita LD, Martien R, Ysrafil Y, Astuti I. Chitosan nanoparticles mediated delivery of miR-106b-5b to breast cancer cell lines MCF-7 and T47D. Int J Appl Pharm. 2021;13(1):129-34.
6. Ysrafil Y, Mus R, Gama NI, Rahmaisyah D, Nur'amalia R. Emerging mutation in SARS-CoV-2 spike: widening distribution over time in different geographic areas. Biomed J. 2021. doi: 10.1016/j.bj.2021.07.003, PMID 34271250.

7. WHO. Coronavirus (COVID-19); 2020. Available from: https://who.sprinklr.com/ [Last accessed on 15 Nov 2021]

8. Singh M, Nagpal M, Singh V, Sharma A, Dhingra GA, Maman P, Puri V. COVID-19: epidemiology, pathogenicity and global updates. Int J App Pharm. 2020;12(5):16-28. doi: 10.22159/ijap.2020v12i5.38439.

9. Horton R. Offline: COVID-19 is not a pandemic. Lancet. 2020;396(10255):874. doi: 10.1016/S0140-6736(20)32000-6, PMID 32979964.

10. Yadav UN, Rayamajhee B, Mistry SK, Parsekar SS, Mishra SK. A syndemic perspective on the management of noncommunicable diseases amid the COVID-19 pandemic in lowand middle-income countries. Front Public Health. 2020;8:508. doi: 10.3389/fpubh.2020.00508, PMID 33102414.

11. Rasheed AM, Fatak DF, Hashim HA, Maulood MF, Kabah KK, Almusawi YA, Abdulamir AS. The therapeutic potential of convalescent plasma therapy on treating critically ill COVID-19 patients residing in respiratory care units in hospitals in Baghdad, Iraq. Infez Med. 2020;28(3):357-66. PMID 32920571.

12. Cheng $\mathrm{Y}$, Wong R, Soo YO, Wong WS, Lee CK, Ng MH, Chan $\mathrm{P}$, Wong KC, Leung CB, Cheng G. Use of convalescent plasma therapy in SARS patients in Hong Kong. Eur J Clin Microbiol Infect Dis. 2005;24(1):44-6. doi: 10.1007/s10096-004-1271-9, PMID 15616839.

13. Sun M, Xu Y, He H, Zhang L, Wang X, Qiu Q, Sun C, Guo Y, Qiu S, Ma K. A potentially effective treatment for COVID-19: A systematic review and meta-analysis of convalescent plasma therapy in treating severe infectious disease. Int J Infect Dis. 2020;98:33446. doi: 10.1016/j.ijid.2020.06.107, PMID 32634589.

14. Wu XX, Gao HN, Wu HB, Peng XM, Ou HL, Li LJ. Successful treatment of avian-origin influenza A (H7N9) infection using convalescent plasma. Int J Infect Dis. 2015;41:3-5. doi: 10.1016/j.ijid.2015.10.009, PMID 26482389.

15. Florescu DF, Kalil AC, Hewlett AL, Schuh AJ, Stroher U, Uyeki TM, Smith PW. Administration of brincidofovir and convalescent plasma in a patient with Ebola virus disease. Clin Infect Dis. 2015;61(6):969-73. doi: 10.1093/cid/civ395, PMID 25991468.

16. Rajendran K, Krishnasamy N, Rangarajan J, Rathinam J, Natarajan M, Ramachandran A. Convalescent plasma transfusion for the treatment of COVID-19: a systematic review. J Med Virol. 2020;92(9):1475-83. doi: 10.1002/ jmv.25961, PMID 32356910.

17. Agarwal A, Mukherjee A, Kumar G, Chatterjee P, Bhatnagar T, Malhotra P, PLACID Trial Collaborators. Convalescent plasma in the management of moderate covid-19 in adults in India: open-label phase II multicentre randomised controlled trial (PLACID Trial). $\mathrm{Br} \quad$ Med J. 2020;371:m3939. doi: 10.1136/bmj.m3939, PMID 33093056.

18. AlQahtani M, Abdulrahman A, Almadani A, Alali SY, Al Zamrooni AM, Hejab AH. Randomized controlled trial of convalescent plasma therapy against standard therapy in patients with severe COVID-19 disease. Sci Rep. 2021;11(1):9927. doi: 10.1038/s41598-02189444-5, PMID 33976287.

19. Avendano Sola C, Ramos Martinez A, Munez Rubio E, Ruiz Antoran B, Malo de Molina R, Torres F. Convalescent plasma for COVID-19: A multicenter, randomized clinical trial. medRxiv 2020:1-15.

20. Bajpai M, Kumar S, Maheshwari A, Chhabra K. Kale P, Gupta A. Efficacy of convalescent plasma therapy compared to fresh frozen plasma in severely ill covid-19 patients: a pilot randomized controlled trial. medRxiv 2020:1-23.

21. Balcells ME, Rojas L, Le Corre N, Martínez Valdebenito C, Ceballos ME, Ferrés M. Early anti-SARS-CoV-2 convalescent plasma in patients admitted for COVID-19: A randomized Phase II clinical trial. medRxiv 2020:1-25.

22. Gharbharan A, Jordans CCE, Geurtsvan Kessel C, den Hollander JG, Karim F, Mollema FPN. Convalescent plasma for COVID-19. A randomized clinical trial. medRxiv 2020:1-16.

23. Li L, Zhang W, Hu Y, Tong X, Zheng S, Yang J, Kong Y, Ren L, Wei Q, Mei H, Hu C, Tao C, Yang R, Wang J, Yu Y, Guo Y, Wu X, Xu Z, Zeng L, Xiong N, Chen L, Wang J, Man N, Liu Y, Xu H, Deng E, 
Zhang X, Li C, Wang C, Su S, Zhang L, Wang J, Wu Y, Liu Z. Effect of convalescent plasma therapy on time to clinical improvement in patients with severe and life-threatening COVID-19: A randomized clinical trial. JAMA. 2020;324(5):46070. doi: 10.1001/jama.2020.10044, PMID 32492084.

24. Duan K, Liu B, Li C, Zhang H, Yu T, Qu J, Zhou M, Chen L, Meng S, $\mathrm{Hu}$ Y, Peng C, Yuan M, Huang J, Wang Z, Yu J, Gao X, Wang D, Yu X, Li L, Zhang J, Wu X, Li B, Xu Y, Chen W, Peng Y, Hu Y, Lin L, Liu X, Huang S, Zhou Z, Zhang L, Wang Y, Zhang Z, Deng K, Xia Z, Gong Q, Zhang W, Zheng X, Liu Y, Yang H, Zhou D, Yu D, Hou J, Shi Z, Chen S, Chen Z, Zhang X, Yang X. Effectiveness of convalescent plasma therapy in severe COVID-19 patients. Proc Natl Acad Sci U S A. 2020;117(17):9490-6. doi: 10.1073/pnas.2004168117, PMID 32253318.

25. Erkurt MA, Sarici A, Berber I, Kuku I, Kaya E, Ozgul M. Lifesaving effect of convalescent plasma treatment in covid-19 disease: clinical trial from eastern Anatolia. Transfus Apher Sci. 2020;59(5):102867. doi: 10.1016/j.transci.2020.102867.

26. Olivares Gazca JC, Priesca Marín JM, Ojeda Laguna M, Garces Eisele J, Soto Olvera S, Palacios Alonso A, Izquierdo Vega J, Chacon Cano R, Arizpe Bravo D, López Trujillo MA, Cantero Fortiz Y, Fernandez Lara D, Ruiz Delgado GJ, Ruiz Arguelles GJ. Infusion of convalescent plasma is associated with clinical improvement in critically ill patients with covid-19: a pilot study. Rev Invest Clin. 2020;72(3):159-64. doi: 10.24875/RIC.20000237, PMID 32584322.

27. Salazar E, Christensen PA, Graviss EA, Nguyen DT, Castillo B, Chen J, Lopez BV, Eagar TN, Yi X, Zhao P, Rogers J, Shehabeldin A, Joseph D, Leveque C, Olsen RJ, Bernard DW, Gollihar J, Musser JM. Treatment of coronavirus Disease 2019 patients with convalescent plasma reveals a signal of significantly decreased mortality. Am J Pathol. 2020;190(11):2290-303. doi: 10.1016/j.ajpath.2020.08.001, PMID 32795424.

28. Ibrahim D, Dulipsingh L, Zapatka L, Eadie R, Crowell R, Williams K, Wakefield DB, Cook L, Puff J, Hussain SA. Factors associated with good patient outcomes following convalescent plasma in COVID-19: A prospective Phase II clinical trial. Infect Dis Ther. 2020;9(4):913-26. doi: 10.1007/s40121-020-003412, PMID 32951151.

29. Abolghasemi H, Eshghi $P$, Cheraghali AM, Imani Fooladi AA, Bolouki Moghaddam F, Imanizadeh S, Moeini Maleki M, Ranjkesh M, Rezapour M, Bahramifar A, Einollahi B, Hosseini MJ, Jafari NJ, Nikpouraghdam M, Sadri N, Tazik M, Sali S, Okati S, Askari E, Tabarsi P, Aslani J, Sharifipour E, Jarahzadeh MH, Khodakarim N, Salesi M, Jafari R, Shahverdi S. Clinical efficacy of convalescent plasma for treatment of COVID-19 infections: results of a multicenter clinical study. Transfus Apher Sci. 2020;59(5):102875. doi: 10.1016/j.transci.2020.102875.
30. Altuntas F, Ata N, Yigenoglu TN, Bascı S, Dal MS, Korkmaz S. Convalescent plasma therapy in patients with COVID-19. Transfus Apher Sci. 2020, PMID 102955.

31. Omrani AS, Zaqout A, Baiou A, Daghfal J, Elkum N, Alattar RA, Bakdach D, Abusriwil H, Mostafa AM, Alhariri B, Ambra N, Khatib M, Eldeeb AM, Merenkov Z, Fawzi Z, Hmissi SM, Hssain AA, Coyle PV, Alsoub H, Almaslamani MA, Alkhal A. Convalescent plasma for the treatment of patients with severe coronavirus disease 2019: A preliminary report. J Med Virol. 2021;93(3):1678-86. doi: 10.1002/jmv.26537, PMID 32965715.

32. Wu Y, Hong K, Ruan L, Yang X, Zhang J, Xu J, Pan S, Ren L, Chen L, Huang C, Shang Y. Patients with prolonged positivity of SARS-CoV-2 RNA benefit from convalescent plasma therapy: A retrospective study. Virol Sin. 2020;35(6):768-75. doi: 10.1007/s12250-020-00281-8, PMID 32865701.

33. Zeng QL, Yu ZJ, Gou JJ, Li GM, Ma SH, Zhang GF, Xu JH, Lin WB, Cui GL, Zhang MM, Li C, Wang ZS, Zhang ZH, Liu ZS. Effect of convalescent plasma therapy on viral shedding and survival in patients with coronavirus Disease 2019. J Infect Dis. 2020;222(1):38-43. doi: 10.1093/infdis/jiaa228, PMID 32348485.

34. Ng KT, Oong XY, Lim SH, Chook JB, Takebe Y, Chan YF, Chan KG, Hanafi NS, Pang YK, Kamarulzaman A, Tee KK. Viral load and sequence analysis reveal the symptom severity, diversity, and transmission clusters of rhinovirus infections. Clin Infect Dis. 2018;67(2):261-8. doi: 10.1093/cid/ciy063, PMID 29385423.

35. Tian X, Li C, Huang A, Xia S, Lu S, Shi Z, Lu L, Jiang S, Yang Z, Wu Y, Ying T. Potent binding of 2019 novel coronavirus spike protein by a SARS coronavirus-specific human monoclonal antibody. Emerg Microbes Infect. 2020;9(1):382-5. doi: 10.1080/22221751.2020.1729069, PMID 32065055.

36. Murphy K, Travers P, Walport M, Janeway C. Janeway's immunobiology. 8th ed. New York: Garland Publishing Science; 2012.

37. French MA, Moodley Y. The role of SARS-CoV-2 antibodies in COVID-19: healing in most, harm at times. Respirology. 2020;25(7):680-2. doi: 10.1111/resp.13852, PMID 32436320.

38. Li L, Tong X, Chen H, He R, Lv Q, Yang R, Zhao L, Wang J, Xu H, Liu C, Chen G, Chen S, Li C, Qiao J, Yang J, Wu Y, Liu Z. Characteristics and serological patterns of COVID-19 convalescent plasma donors: optimal donors and timing of donation. Transfusion. 2020;60(8):1765-72. doi: 10.1111/trf.15918, PMID 32627216.

39. Rojas M, Rodriguez Y, Monsalve DM, Acosta-Ampudia Y, Camacho B, Gallo JE, Rojas Villarraga A, Ramirez Santana C, Díaz Coronado JC, Manrique R, Mantilla RD, Shoenfeld Y, Anaya JM. Convalescent plasma in covid-19: possible mechanisms of action. Autoimmun Rev. 2020;19(7):102554. doi: 10.1016/j.autrev.2020.102554. 